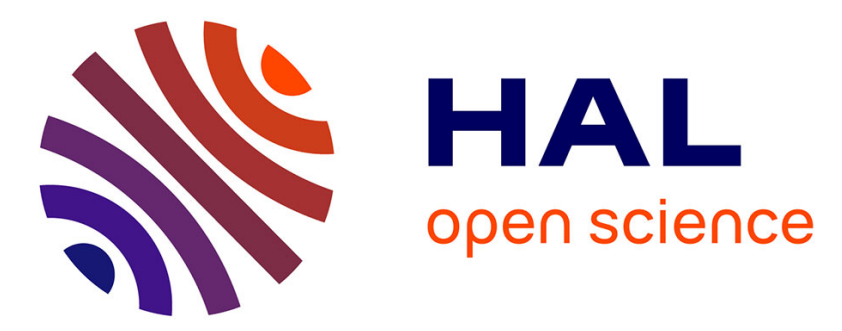

\title{
Efficient target tracking for 3D ultrasound-guided needle steering
}

Guillaume Lapouge, Gaelle Fiard, Philippe Poignet, Jocelyne Troccaz

\section{To cite this version:}

Guillaume Lapouge, Gaelle Fiard, Philippe Poignet, Jocelyne Troccaz. Efficient target tracking for 3D ultrasound-guided needle steering. SPIE Medical Imaging, Feb 2020, Houston, United States. pp.\#113150I, 10.1117/12.2548321 . hal-02498231

\section{HAL Id: hal-02498231 \\ https://hal.science/hal-02498231}

Submitted on 4 Mar 2020

HAL is a multi-disciplinary open access archive for the deposit and dissemination of scientific research documents, whether they are published or not. The documents may come from teaching and research institutions in France or abroad, or from public or private research centers.
L'archive ouverte pluridisciplinaire HAL, est destinée au dépôt et à la diffusion de documents scientifiques de niveau recherche, publiés ou non, émanant des établissements d'enseignement et de recherche français ou étrangers, des laboratoires publics ou privés. 


\title{
Efficient target tracking for 3D ultrasound-guided needle steering
}

\author{
Guillaume Lapouge* ${ }^{\mathrm{a}, \mathrm{b}}$, Gaelle Fiard $^{\mathrm{c}}$, Philippe Poignet ${ }^{\mathrm{b}}$, Jocelyne Troccaz $^{\mathrm{a}}$ \\ aIMC-IMAG, Univ. Grenoble Alpes, CNRS, Grenoble INP, F-38000 Grenoble, France \\ ${ }^{\mathrm{b}}$ LIRMM, Univ. Montpellier, CNRS, F-34090 Montpellier, France \\ ${ }^{c}$ Department of Urology, Grenoble Alpes University Hospital, Grenoble, France
}

\begin{abstract}
3D ultrasound imaging can be used in the context of robotic needle steering to reach a physical target with a flexible, steerable needle. During the insertion, the tissue may be deformed by the inserted needle, patient breathing or external force application. It may therefore be necessary to track intra-operatively the displacement of the target. Most ultrasound based needle steering works concentrate on 2D ultrasound probes [1], [2], [3] which do not allow to simultaneously track both the target and the needle during 3D needle steering. Physical target tracking in 3D ultrasound-guided needle steering is seldom carried out [4][5], and may require computational power that is precious for intra-operative needle steering.

This paper proposes a new approach for computationally inexpensive and precise tracking of a moving target in 3D Bmode ultrasound volumes. It is based on the interconnection of intensity-based tracking and motion estimation algorithms. The intensity-based tracking consists in a 3D extension of the Diamond Shape block matching algorithm, used here for the first time in 3D ultrasound volumes for tissue tracking. The motion estimation is done by linear Kalman filtering. It predicts the next target position and ensures faster and more robust convergence of the Diamond Shape block matching algorithm. An experimental validation on ex-vivo tissue is proposed with promising tracking precision (estimated average error of $0.3 \mathrm{~mm}$ ) while significantly lowering the computational cost when compared to classical block matching based tracking.
\end{abstract}

Keywords: Target Tracking, 3D Diamond Shape Block Matching, 3D Ultrasound, Needle Steering

\section{INTRODUCTION}

A percutaneous procedure consists in inserting a needle through a patient skin to reach a specific area. It can be done to deliver a drug, do radio ablation and realize a biopsy among others. The operation is often carried out under B-mode ultrasound imaging which provides a relatively inexpensive and convenient image feedback. However, the low quality of such imaging requires a strong effort of mental reconstruction from the surgeon. Besides, as it is inserted, the needle may bend and the tissue might undergo some displacement, potentially making the surgeon miss a moving target. To tackle these issues, robotic needle steering has been introduced, using among other techniques the asymmetry of the needle tip to follow complex trajectories and correct the needle insertion. However, as the target may be mobile, intra-operative target tracking is necessary for a clinical application.

Most ultrasound (US) based needle steering works concentrate on 2D US probes [1], [2], [3]. This does not allow to simultaneously track both the target and the needle during 3D needle steering in the images. In [6], a model based prediction is proposed to overcome this limitation for simple phantom translations. Target tracking in 3D US-guided needle steering is rarely realized. In [4], 3D target tracking is achieved with a Star algorithm applied to 2D slices, for physical, sphere shaped targets. In [5], an ex-vivo tissue patch is tracked using exhaustive search block matching. However, in 3D US volumes, tissue tracking may present a computational complexity incompatible with the real-time needs of needle steering [7], [8].

This paper proposes the interconnection of intensity-based tracking and motion estimation algorithms for computationally inexpensive 3D target tracking in 3D B-mode US volumes. It is based on a $3 \mathrm{D}$ extension of the Diamond Shape block matching algorithm, used here for the first time in 3D ultrasound volumes for tissue tracking. The temporal continuity of the target motion is taken into account through state observation. The proposed solution allows for large target displacement tracking while being compatible with 3D US-guided needle steering. Its performance and limitations are detailed below. 
*Corresponding author: guillaume.lapouge@univ-grenoble-alpes.fr

\section{MATERIALS AND METHODS}

\subsection{System overview}

The proposed target tracking algorithm is part of a complete system developed to achieve needle steering. It can be subdivided into interconnected blocks as illustrated in Fig.1.
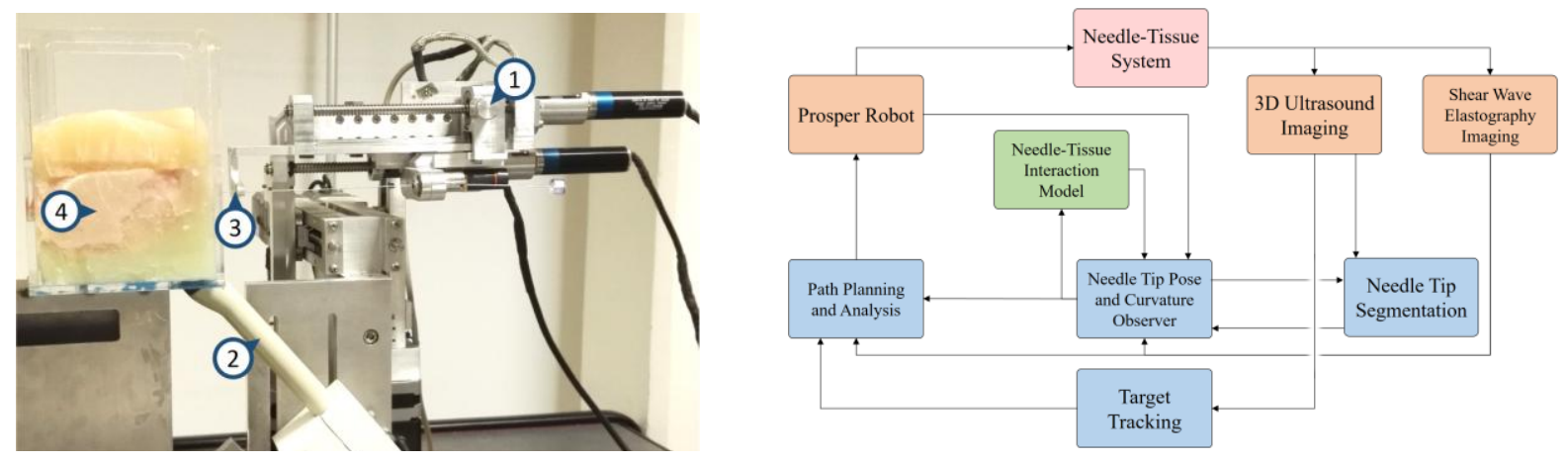

1 - PROSPER Robot; 2 - 3D US end-fire probe; 3 - Needle; 4 - Tissue.

Figure 1: Needle steering system overview [9].

This system uses the asymmetry of a flexible needle tip to deflect the needle and achieve 3D needle steering guided under 3D B-mode US feedback. It features needle tracking algorithms detailed in [9] and path planning algorithm that is not described in depth in this work. The path planning algorithm recomputes a possible path to the target every second and adapt to the new state of the system. In this context, if a physical target is tracked online, the system adapts to its movement and reaches it if possible.

\subsection{Target Tracking}

In our previous work [5], we proposed a simple block matching approach for target tracking. Although the precision performance was satisfying, the solution was computationally expensive. Parallelized on a GPU with the Nvidiaß CUDA® library, one iteration of this algorithm took $150 \mathrm{~ms}$, using a block size of $5 \times 5 \times 5 \mathrm{~mm}(13 \times 13 \times 13$ voxels $)$ and a searching area of $10 \times 10 \times 10 \mathrm{~mm}(25 \times 25 \times 25$ voxels $)$. Besides, any movement greater that the user-set size of the searching area would not be detected and provoke a failure of the algorithm. We propose another approach for target tracking based on the interconnection of intensity-based tracking and motion estimation algorithms (see Fig. 2). This solution helps reduce the computational cost of image processing by accounting for motion prediction information.

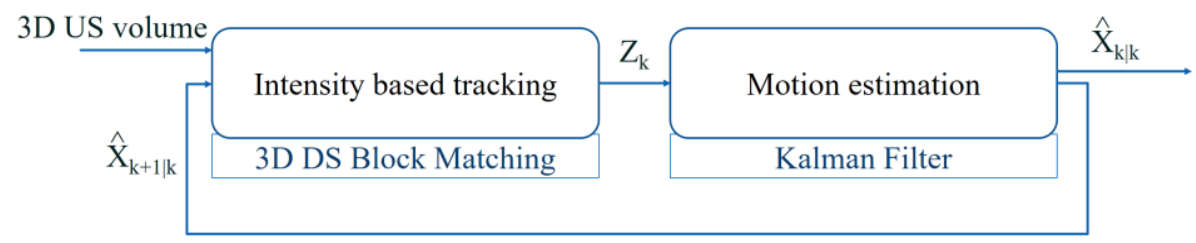

Figure 2: Proposed target tracking.

\subsubsection{Motion estimation}

Under the assumption that there is no measurable input directly related to the target motion, only the hypothesis of motion smoothness is kept. In such case, we assume that the system can be modeled by the state transition equations:

$$
\text { With } \quad F=\left[\begin{array}{cccccc}
1 & 0 & 0 & \delta t & 0 & 0 \\
0 & 1 & 0 & 0 & \delta t & 0 \\
0 & 0 & 1 & 0 & 0 & \delta t \\
n & n & n & 1 & n & n
\end{array}\right] \quad \text { and }
$$




$$
Z_{k}=H X_{k}+v_{k}
$$

$$
H=\left[\begin{array}{llllll}
1 & 0 & 0 & 0 & 0 & 0 \\
0 & 1 & 0 & 0 & 0 & 0 \\
0 & 0 & 1 & 0 & 0 & 0
\end{array}\right]
$$

where $X_{k}=\left[\begin{array}{llllll}x_{k} & y_{k} & z_{k} & \dot{x_{k}} & \dot{y_{k}} & \dot{z_{k}}\end{array}\right]^{T}$ is the state vector composed of the target Cartesian position and velocities, $w_{k}$ is an additive system noise. $Z_{k}$ is the measurement vector of the target Cartesian position provided by the intensitybased tracking algorithm. $v_{k}$ is an additive measurement noise.

A Kalman filter estimates the state $\hat{X}_{k \mid k}$ and predicts $\hat{X}_{k+1 \mid k}=F \hat{X}_{k \mid k} . \quad \hat{X}_{k \mid k}$ is considered as the current estimated target state while $\widehat{X}_{k+1 \mid k}$ is sent to the intensity-based tracking algorithm as an a priori knowledge of the future target Cartesian position. In this way, the motion estimation algorithm has two distinct uses: to filter noisy data and to predict the next target position for faster image processing. The Kalman filter is initialized such that $\hat{X}_{0 \mid 0}$ is composed of the initial target position with null velocities. The covariance matrix of the observation noise $R=\sigma_{R}^{2} I_{3}$ is set so that the noise standard deviation $\sigma_{R}=0.4 \mathrm{~mm}$ reflects the resolution of the chosen ultrasound probe. For more simplicity, the covariance matrix of the process noise can be chosen as $Q=\alpha I_{6}$. Where $\alpha$ can be tuned depending on the application, reflecting how important unmodeled uncertainties affect (1) (in this paper $\alpha=0.1$, arbitrarily chosen). The error covariance matrix $\mathrm{P}$ is classically set as the identity matrix.

\subsubsection{Intensity-based tracking}

In this work, we propose a 3D extension of the Diamond Shape block matching (DS) [10] as the intensity-based tracking algorithm (see Fig. 3). Indeed, it is significantly less computationally expensive than Exhaustive Search (ES) block matching while having similar peak signal-to-noise ratio [11]. The choice of intensity-based tracking over feature-based tracking can be explained by the absence of reliable features to track in small 3D US volumes samples. Feature-based tracking seems therefore more adapted to track displacements when given access to the whole 3D US volume data. Such strategy would require the analysis of a large amount of data which is incompatible with the real-time needs of needle steering.

The 3D DS algorithm functions as follows. A block $\mathrm{B}_{\text {target }}$, centered around the target, is defined in a reference 3D US volume. The block with Minimum Block Distortion (MBD) (i.e. the one with minimal Sum of Squared Distances (SSD) with $\mathrm{B}_{\text {target }}$ ) is then searched for in subsequent US volumes. To do so, the SSD is computed between $\mathrm{B}_{\text {target }}$ and blocks centered on the 19 points of the Large Diamond Search Pattern (LDSP) (in blue in Fig. 3(a)). The LDSP is then translated to the MBD point and the point checking starts again. If the MBD point is a diamond corner (resp. an edge) point, only 13 (resp. 9) points need to be checked (see Fig. 3(b)). This is due to the overlapping of checking points that naturally occur when the search pattern is displaced. If the MBD point is the center point, the pattern is switched to the Small Diamond Search Pattern (SDSP) (in red in Fig. 3(a)) and the best MBD point is taken as the result of the 3D DS algorithm. In the case where the algorithm does not find the best MBD point after $\mathrm{N}$ trials ( $\mathrm{N}$ set by the user), or if the $\mathrm{SSD}$ between the converged block and $\mathrm{B}_{\text {target }}$ is superior to $\mathrm{SSD}_{\text {threshold, }}$, the block matching is considered as failed.
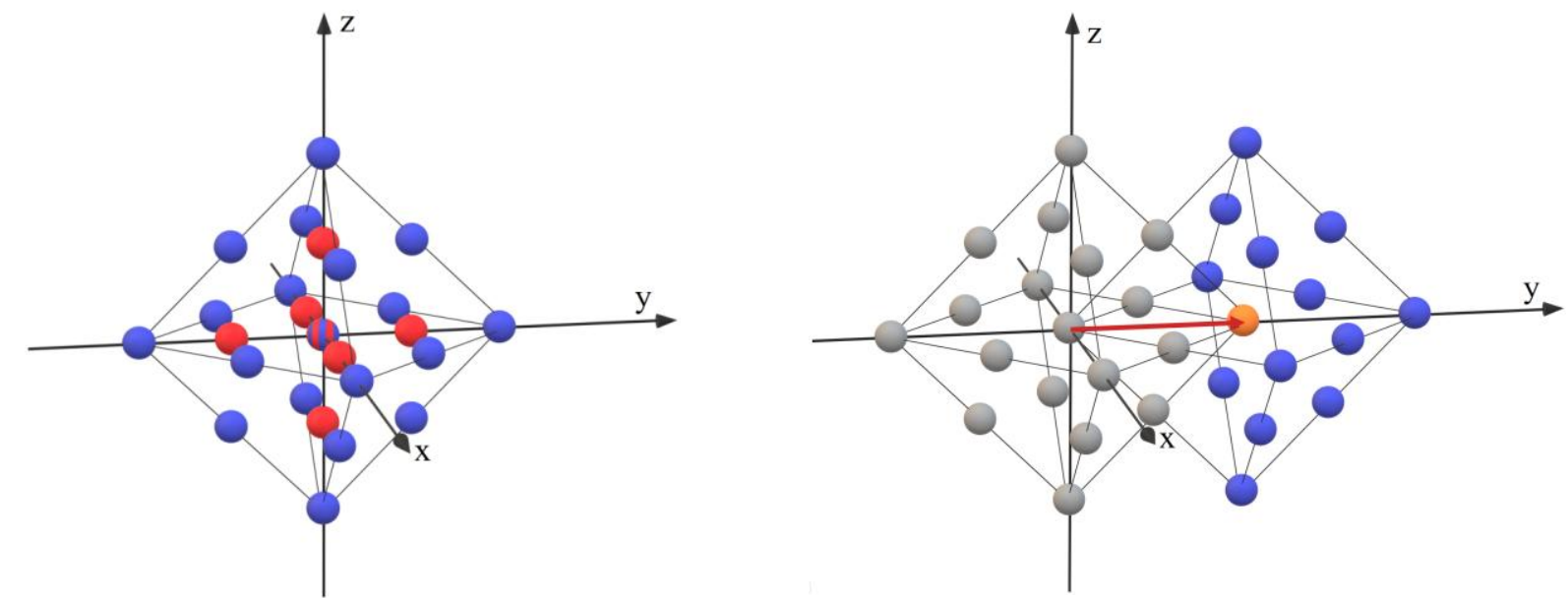


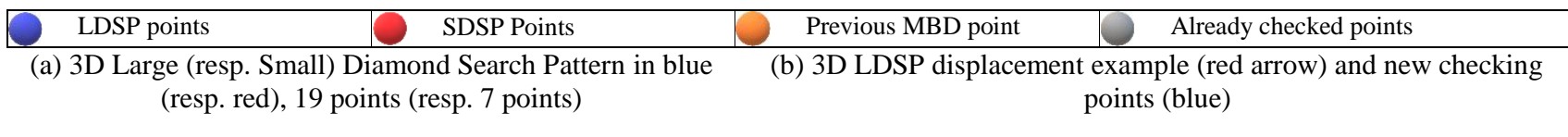

Figure 3: 3D Diamond search algorithm checking points.

\subsubsection{Workflow}

The workflow is designed as follows. A target is set by the user in the $\mathrm{k}^{\text {th }} 3 \mathrm{D}$ US volume. A cubic block $\mathrm{B}_{\text {target, }}$ of edge size $n$ and centered around the target location is registered as reference block. The Kalman filter is initialized with the Cartesian position of $\mathrm{B}_{\text {target }}$ and with null velocities. At the reception of the $\mathrm{k}+1^{\text {th }} 3 \mathrm{D}$ US volume, the $3 \mathrm{D}$ DS algorithm is then started from the predicted Cartesian position in $\hat{X}_{k+1 \mid k}$. If the algorithm converges, the measurement vector $Z_{k+1}$ is updated and sent to the Kalman filter. Else, the algorithm waits for the next US frame. The estimation $\widehat{X}_{k+1 \mid k+1}$ is sent to the GUI and path planning algorithm which adapts the robot movement to the newly estimated target position. At the reception of the $\mathrm{k}+2^{\text {th }} 3 \mathrm{D}$ US volume, the 3D DS algorithm is then started from the predicted Cartesian position in $\hat{X}_{k+2 \mid k+1}$. And the whole process starts again.

\section{EXPERIMENTAL ASSESSMENT}

The proposed solution has been developed in C++ in the CamiTK framework (see [12] for more details). On a laptop with an Intel ${ }^{\circledR}$ Core ${ }^{\mathrm{TM}} \mathrm{I} 5-8300 \mathrm{H}$ CPU, target tracking takes an average time of $2.9 \mathrm{~ms}$, using a block size of $8 \times 8 \times 8 \mathrm{~mm}$ (20x20x20 voxels) and a maximum number of LDSP iterations of 10. Compared to our previous work mentioned at the beginning of 2.2, the algorithm realizes up to 66 times less MBD computations in the chosen scenario. The US volumes are acquired at $1 \mathrm{~Hz}$ with a 3D endorectal end-fire probe 4DEC-9/10 used with the Ultrasonix Sonix RP ultrasound system. The US volume voxels are cubes with $0.37 \mathrm{~mm}$ edges.

\subsection{Ex-vivo chicken tissue translation}

The validation of the algorithm has been made on a phantom with ex-vivo chicken breast tissue samples enclosed in agar. The phantom was fixed to a XY motorized translation table realizing a precise circular motion with $5 \mathrm{~mm}$ radius. The US probe was stationary, fixed on top of the moving phantom and in constant direct contact with it (see Fig. 4). The resulting motion of the tissue, as perceived by the probe, is a circle in the XZ plane in the US volume frame. Because we aim to validate our method with submillimeter accuracy, we analyze the relative motion of the target and fit the best circle to its tracked motion. From this best fit, two metrics are computed:

- The shape error of the circular motion (mean distance of the estimated path to the best-fit circle).

- The radius error of the circular motion (the absolute value of the difference between the best-fit circle radius and the $5 \mathrm{~mm}$ expected). 


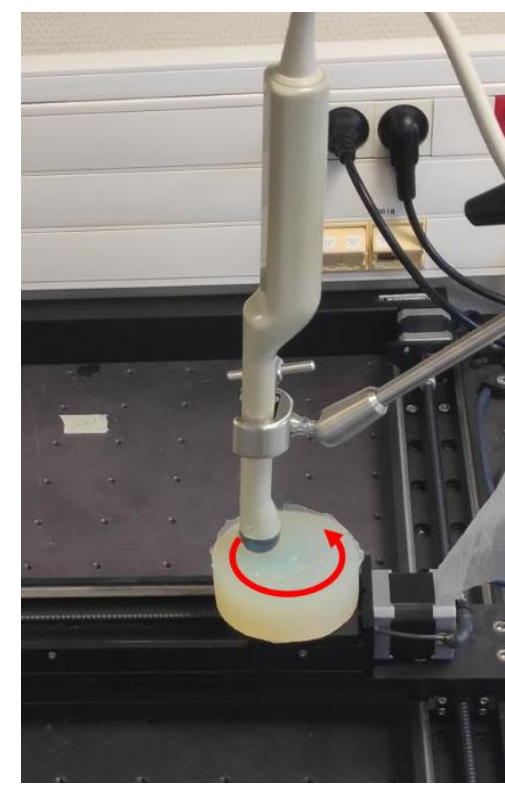

Figure 4: Experimental setup for circular translation trials. For better visualization, the phantom in this picture is made of silicone.

\subsection{Needle steering in cadaveric tissue}

In order to validate the functioning of this algorithm with clinically compatible needle steering 10 insertions were made into the prostate of a cadaver through the perineum. The body is set in the classic brachytherapy gynecologic position (see Fig. 5(a)). The prostate and the surrounding tissue are observed with the probe presented in subsection 3.1. Before the insertion, 4 standard brachytherapy seeds (cylinders of diameter $0.8 \mathrm{~mm}$ and length $4.5 \mathrm{~mm}$ ) were implanted in the prostate to provide physical targets to track. The tissue deformation occurs when the needle is inserted into the prostate, the tracking is either done on a brachytherapy seed or some calcification in the prostate (see Fig. 5(b)). This way, the automatic target tracking can be compared to a manual tracking realized by a non-expert user.

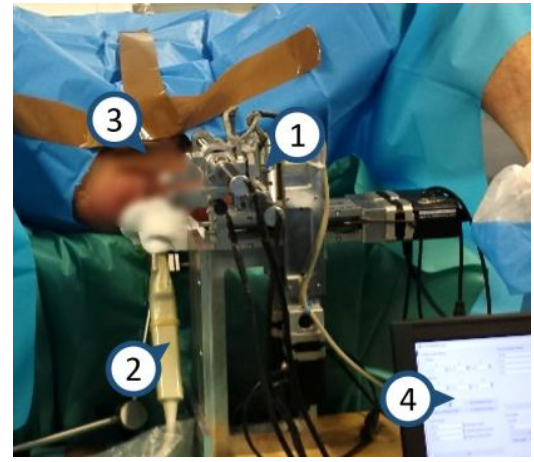

(a) Experimental setup for cadaver trials. 1 - PROSPER Robot; 2 - 3D US end-fire probe; 3 - Perineum; 4 Laptop running needle steering and target tracking algorithms.

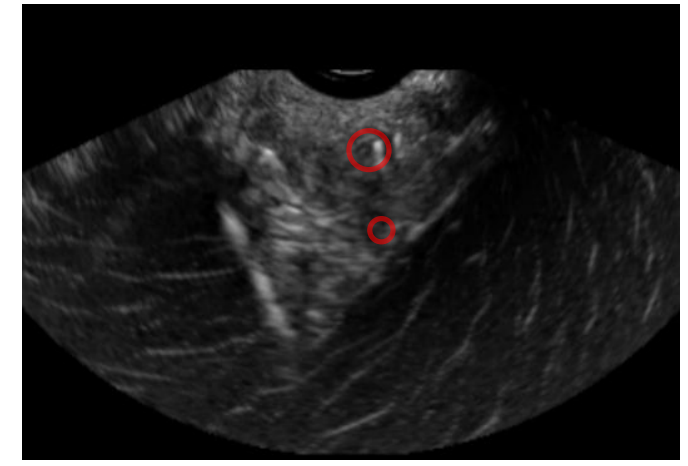

(b) Corresponding 3D US volume. Brachytherapy seed (large red circle) and calcification (small red circle).

Figure 5: Experimental validation through cadaveric trials.

\section{RESULTS}

The results presented below are part of a set of experiments that are not all presented in this paper. A video detailing the entirety of the experimental validation is available at https://youtu.be/jwlGTxvzkEM. 


\subsection{Ex-vivo chicken tissue translation}

The target tracking was tested on 207 different locations in the 3D US volume. An example of target tracking is provided in Fig. 6.
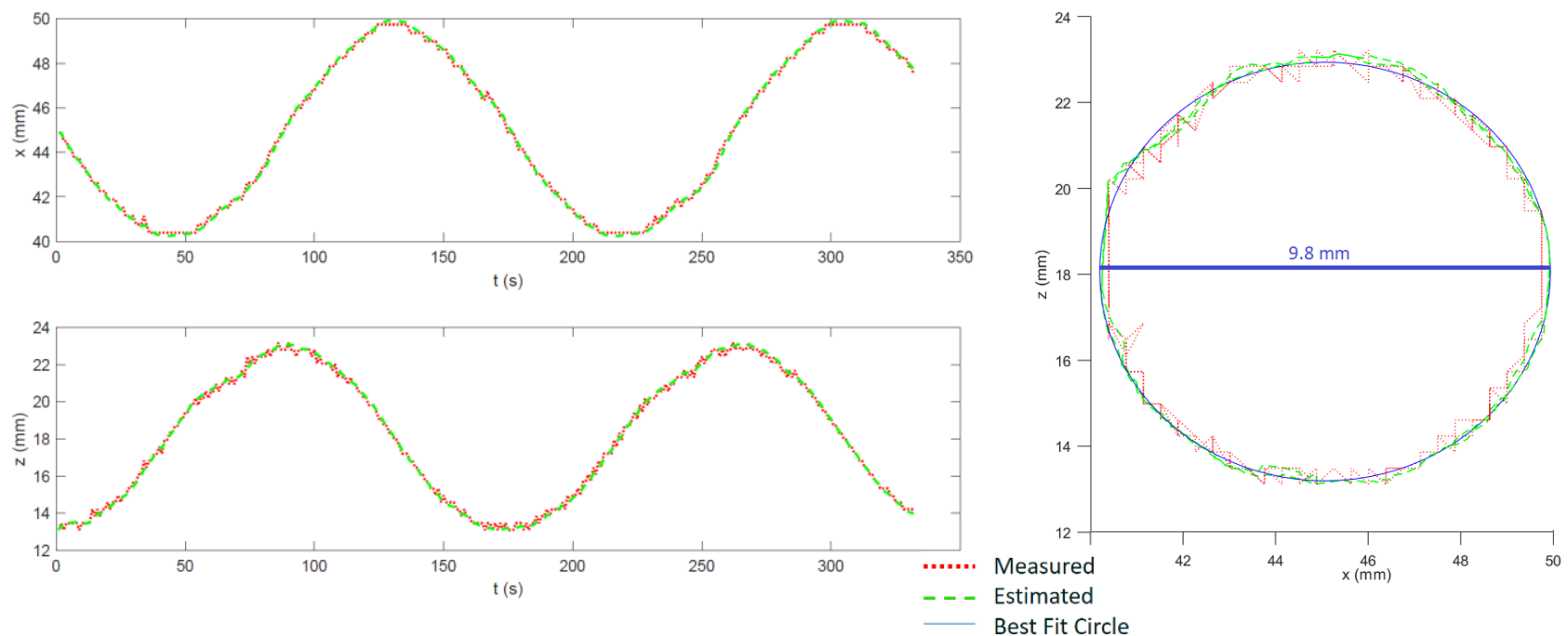

Figure 6: Example of 3D target tracking in ex-vivo chicken tissue.

The usefulness of the Kalman filter can be seen as it filters eventual mechanical jitter of the probe, appearing as an oscillation of the US volume on the $\mathrm{z}$ axis. It also smoothens the estimated target trajectory. Besides, using the prediction of the future target position as starting search point has reduced the computational cost up to $20 \%$ in such scenario. This trend becomes more obvious as the displacement speed of the target increases. The shape and radius errors are reported in Fig. 7. Specific regions with bad tracking performance can be explained by the presence of artefacts, shadowing and saturation (yellow, orange and white ellipses in Fig. 7(a)). The agar region of the phantom shows good circular motion estimation with a constant error in radius while the chicken tissue shows good circular motion estimation with good radius. This may be due to the difference in propagation speed of the US waves in the phantom and in the tissue causing a shift in proportions. We evaluated this algorithm on 121 points of a phantom with chicken only, where the shadowing and saturation regions are not taken into account. The tracking performance, detailed in Fig 7(b), is characterized by an average shape error of $0.3 \mathrm{~mm}$. There are many starting points with poor tracking results, due to the lack of proper structure to track.
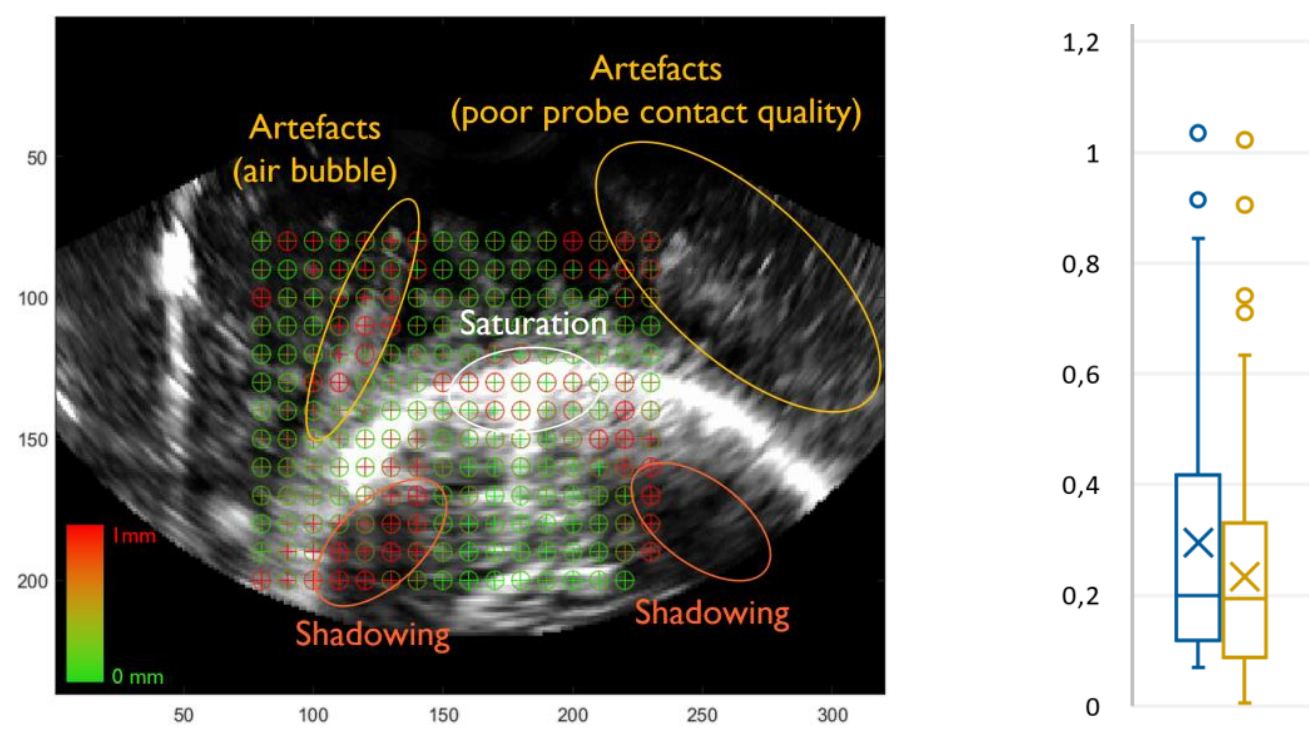
Error, shape of the circular motion

Error, radius of the circular motion

(a) Visual representation of the target tracking precision at various starting locations. A green color corresponds to a small error; a red color corresponds to an error superior to $1 \mathrm{~mm}$ as indicated in the color code.
Shape error ( $\mathrm{mm})$

Radius error ( $\mathrm{mm}$ )

(b) Detail in chicken (cropped). The cross indicates the mean value, the line the median value.

Figure 7: 3D target tracking results for ex-vivo chicken tissue (lower part in (a)) in an agar phantom (upper part in (a)).

\subsection{Needle steering in cadaveric tissue}

For a needle insertion, the brachytherapy seed and a calcification have been tracked both manually and automatically. 20 manual segmentations are compared to the corresponding automatic tracking results. The performance results are reported in Table 1. The results show an average difference around $0.3 \mathrm{~mm}$ and $0.4 \mathrm{~mm}$ for seed and calcification tracking respectively. The precision of the manual segmentation itself being close to $0.3 \mathrm{~mm}$, these results are promising. The difference in accuracy can be explained by the increased visibility and stability in appearance of the brachytherapy seed compared to the calcification. This makes it harder to segment both automatically and manually. These observations add to those made in subsection 4.1, demonstrating the importance of the initial target choice.

Table 1. Difference between manual and automatic tracking during needle steering in a cadaver prostate.

\begin{tabular}{|l|c|c|c|c|}
\hline Tracked object & $\mathbf{\Delta x}(\mathbf{m m})$ & $\Delta \mathbf{y}(\mathbf{m m})$ & $\Delta \mathbf{z}(\mathbf{m m})$ & $\boldsymbol{\Delta}(\mathbf{m m})$ \\
\hline \multirow{2}{*}{ Brachytherapy seed } & $0.18 \pm 0.10$ & $0.11 \pm 0.07$ & $0.17 \pm 0.13$ & $0.29 \pm 0.13$ \\
& {$[0.03 ; 0.32]$} & {$[0.04 ; 0.29]$} & {$[0.01 ; 0.45]$} & {$[0.12 ; 0.49]$} \\
\hline \multirow{2}{*}{ Calcification } & $0.18 \pm 0.12$ & $0.16 \pm 0.17$ & $0.22 \pm 0.19$ & $0.39 \pm 0.16$ \\
& {$[0.01 ; 0.35]$} & {$[0.02 ; 0.61]$} & {$[0.04 ; 0.60]$} & {$[0.21 ; 0.66]$} \\
\hline
\end{tabular}

\section{CONCLUSION}

We have proposed a real-time compatible target tracking algorithm validated on cadaveric 3D US volumes. The performance of the tracking is promising, achieving good precision in trackable zones. The user should be aware of strong artefacts zones in which the proposed solution may fail. The algorithm greatly decreases the computational costs when compared to exhaustive search block matching. The interconnection between motion estimation and image processing limits eventual local extrema convergence and allows for large tissue movement. Besides, using the prediction of the future target position as starting search point further reduces the computational cost. However, for high velocity and changing motion direction, the algorithm may not converge as the predicted search point may significantly vary from the real target position. The validation proposed here for small deformations is not valid in case of large tissue distortion. Indeed, in such instance, the blocks appearance changes significantly and no correspondence can be done using block matching. In the context of needle steering, such a case is unlikely.

\section{ACKNOWLEDGEMENTS}

We would like to thank the Laboratoire d'Anatomie Des Alpes Françaises for their kind help to realize the cadaver trials. This work was partly supported by the Investissements d'Avenir programme (Labex CAMI) under reference ANR-11LABX-0004.

\section{REFERENCES}

[1] M. Abayazid, P. Moreira, N. Shahriari, A. Zompas, et S. Misra, "Three-Dimensional Needle Steering Using Automated Breast Volume Scanner (ABVS)", Journal of Medical Robotics Research 1(01), 1640005 (2016).

[2] B. Fallahi, C. Rossa, R. S. Sloboda, N. Usmani, et M. Tavakoli, "Sliding-based image-guided 3D needle steering in soft tissue", Control Engineering Practice 63, 34- 43 (2017). 
[3] J. Carriere, M. Khadem, C. Rossa, N. Usmani, R. Sloboda, et M. Tavakoli, "Event-Triggered 3D Needle Control Using a Reduced-Order Computationally Efficient Bicycle Model in a Constrained Optimization Framework", Journal of Medical Robotics Research 4(01), 1842004 (2018).

[4] J. Chevrie, A. Krupa, et M. Babel, "Real-time Teleoperation of Flexible Beveled-tip Needle Insertion using Haptic Force Feedback and 3D Ultrasound Guidance", IEEE International Conference on Robotics and Automation, (2019).

[5] P. Mignon, P. Poignet, et J. Troccaz, “Automatic Robotic Steering of Flexible Needles from 3D Ultrasound Images in Phantoms and Ex Vivo Biological Tissue", Annals of Biomedical Engineering 46(09), 1385-1396 (2018).

[6] P. Moreira, M. Abayazid, et S. Misra, "Towards physiological motion compensation for flexible needle interventions", IEEE/RSJ International Conference on Intelligent Robots and Systems (IROS), 831-836 (2015).

[7] L. Royer, "Real-time tracking of deformable targets in 3D ultrasound sequences", Medical Imaging INSA de Rennes, (2016).

[8] J. Banerjee, C. Klink, E. D. Peters, W. J. Niessen, A. Moelker, et T. van Walsum, "Fast and robust 3D ultrasound registration - Block and game theoretic matching", Medical Image Analysis 20(01), 173- 183 (2015).

[9] G. Lapouge, J. Troccaz, et P. Poignet, "Multi-rate unscented Kalman filtering for pose and curvature estimation in 3D ultrasound-guided needle steering", Control Engineering Practice 80, 116- 124 (2018).

[10] S. Zhu et K.-K. Ma, “A New Diamond Search Algorithm for Fast Block-Matching Motion Estimation”, IEEE transactions on image processing 9(02), 287-290 (2000).

[11] A. Barjatya, "Block Matching Algorithms for Motion Estimation", IEEE Transactions Evolution Computation 8(03), 225-239 (2004).

[12] C. Fouard, A. Deram, Y. Keraval, et E. Promayon, "CamiTK: A Modular Framework Integrating Visualization, Image Processing and Biomechanical Modeling”, Soft Tissue Biomechanical Modeling for Computer Assisted Surgery 11, 323- 354 (2012). 